\title{
A 137-Year History of the Summer Avian Community at the Winous Point Marsh, Port Clinton, Ohio, USA
}

\author{
BRENDAN T. SHIRKEY' , JOHN W. SIMPSON, MICHAEL A. PICCIUTO, Winous Point Marsh Conservancy, Port Clinton, OH, USA.
}

ABSTRACT. The Winous Point Marsh Conservancy and the Winous Point Shooting Club, with landholdings in both Sandusky and Ottawa Counties, Ohio, has completed a survey of the summer resident bird population on the property 4 times spanning 137 years. Although achieving a true census is unrealistic, these surveys have provided a unique, long-term history of changes in the avian community in northwest Ohio since the 1880 s. Surveys were completed in 1880,1930,1960, and 2017. Draining and deforestation of the wetlands in northwest Ohio, by the late 1870s, resulted in the loss of many forested-wetland dependent species such as Blue-gray Gnatcatcher (Polioptila caerulea), Solitary Sandpiper (Tringa solitaria), and Northern Waterthrush (Parkesianoveboracensis) after the 1880 survey. Intensification of agricultural practices after the 1930 survey likely resulted in the loss of the early successional habitat that was associated with smaller, less intensive, agricultural practices and consequently the loss of many grassland nesting species such as Bobolink (Dolichonyx oryzivorus), Eastern Meadowlark (Sturnella magna), Northern Bobwhite (Colinus virginianus), Blue-winged Teal (Anas discors), Northern Pintail (Anas acuta), and Northern Shoveler (Anas clypeata). Several new species were documented during the 2017 survey including Osprey (Pandion haliaetus), Sandhill Crane (Antigone canadensis), Trumpeter Swan (Cygnus buccinator), and American White Pelican (Pelecanus erythrorhynchos). This long-term dataset provides a unique opportunity to investigate the avian immigration, extirpation, and recolonization of a specific site over the past 137 years, providing insight into how landscape-level habitat changes affected the avian community.

\section{INTRODUCTION}

Some of the most consistent and longest-running wildlife monitoring programs in North America, including the North American Breeding Bird Survey (established in 1966) and the Waterfowl Breeding Population and HabitatSurvey (established in 1955), are available to avian biologists and managers (Smith 1995; Sauer et al. 2013). Furthermore, The Ohio Breeding Bird Atlas, describing the distribution of breeding birds across Ohio was completed from 1982 to 1987 (Peterjohn and Rice 1991); a follow-up volume, The Second Atlas of Breeding Birds in Ohio, was undertaken from 2006 to 2011 (Rodewald et al. 2016). Long-term biological monitoring datasets are rare, but can be valuable tools for documenting extirpation, colonization, or even long-term population trends of specific species (Franklin 1989). Additionally, population trend data, or even presence/absence data, can ultimately be linked to landscape-level habitat or environmental changes (Ball et al. 2005; Wintle and Bardos 2006). Although on a much smaller spatial

${ }^{1}$ Address correspondence to Brendan T. Shirkey, Winous Point Marsh Conservancy, 3500 S. Lattimore Road, Port Clinton, OH 43452, USA. Email: brendan@winous.org scale, Ohio also has a unique bird survey with data collection dating back to 1880 .

The Winous Point Marsh Conservancy (WPMC) in Ottawa and Sandusky Counties, Ohio, United States, was formed in 1999 to further the research and conservation interests of the Winous Point Shooting Club, a historic duck hunting club that has owned and managed approximately 900 hectares of Lake Erie coastal marsh since 1856 (Fig.). On 4 occasions in the history of the organization, experienced naturalists have attempted to survey the summer resident bird species on the property (Langdon 1880; Christy 1931; Anderson 1960). This dataset offers a unique opportunity to investigate changes in the avian community of northwest Ohio over 100 years. Although not a true census, these summer surveys form a dataset spanning 137 years that can be used to illustrate changes in the avian community and the extirpation, immigration, and recolonization of the site by various bird species. This report surmises that many of the changes documented in the avian

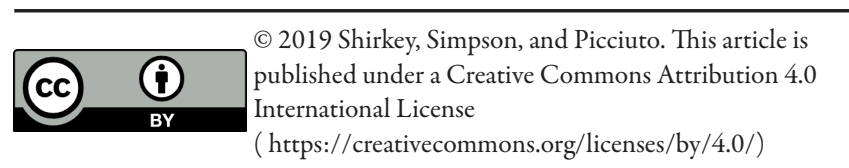


community have coincided with the landscape-level habitat changes that have occurred in northwest Ohio since 1880 (e.g., deforestation, wetland degradation and loss, conversion of grassland, and early successional habitat to agriculture).

\section{Study Area}

\section{METHODS}

Ohio has lost in excess of $90 \%$ of its historical wetland habitat, and much of the habitat that remains has been highly degraded by anthropogenic influences (e.g., increased sediment runoff, nutrient runoff, and invasive species) (Campbell et al. 1995; Wilson and Mitsch 1996). Much of this loss occurred in the coastal marshes of northwestern Ohio. Now only approximately 12,000 hectares of wetlands remain in an area that once consisted of approximately 405,000 hectares and was described as the "Great Black Swamp." The latter covered parts of Lucas, Ottawa, Sandusky, Seneca, Wood, Hancock, Henry, Putnam, Allen, Defiance, Paulding, and Van Wert Counties in Ohio, and Allen County in Indiana (Campbell et al. 1995). The remaining wetlands are often hydrologically disconnected from Lake Erie by necessity to prevent further erosion of wetland vegetation (Andrews 1952; Sherman et al. 1996). Consequently, these wetlands do not function ecologically as they would have historically by providing an expanse of shallow water wetland habitat directly connected to Lake Erie.

WPMC is a privately-owned coastal wetland complex situated at the confluence of the Sandusky River, Muddy Creek, Green Creek, South Creek, and Lake Erie. WPMC landholdings consist of approximately 400 hectares of emergent marsh, 100 hectares of forested wetland, and 400 hectares of open water (Fig.). Emergent marsh habitat is dominated by perennial species such as cattail (Typha spp.), bulrush (Schoenoplectus spp. and Scirpus spp.), burreed (Sparganium spp.), and phragmites (Phragmites australis), as well as annuals such as smartweed (Polygonum spp.), millet (Echinochloa spp.), and nutsedge (Cyperus spp.). Forested wetland habitat is dominated by cottonwood (Populus deltoides), willow (Salix spp.), hickory (Carya spp.), and swamp white oak (Quercus bicolor). Open water habitat owned by

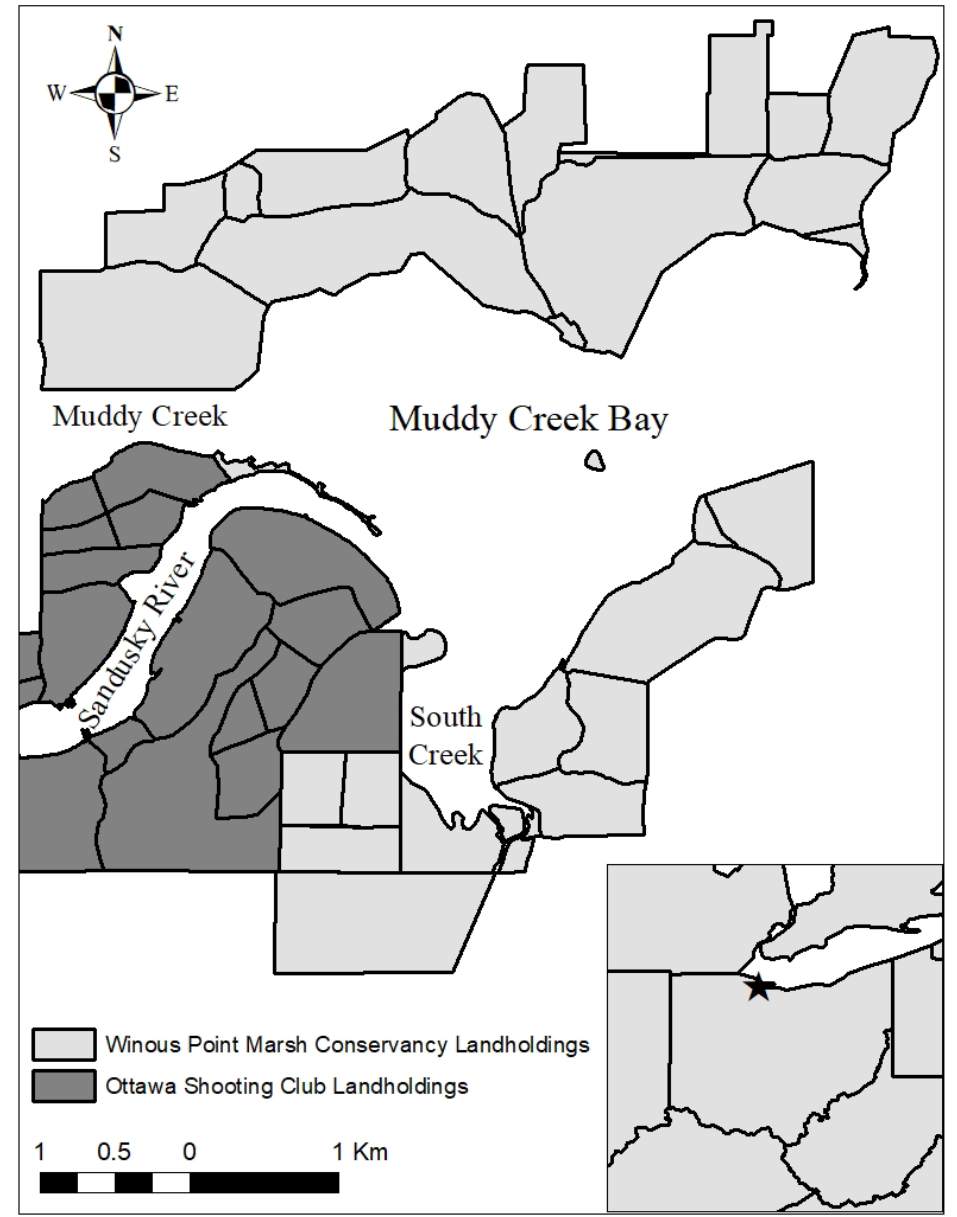

FIGURE. Map with inset showing the areal location of the Winous Point Marsh Conservancy and WPMC property boundaries as well as the current levee system (black outlines) 
WPMC, but outside of the current levee system, is generally devoid of plant life due to turbidity and wave action. During the earliest survey period, however, this habitat type was dominated by large stands of bulrush and wild rice (Zizania spp.) in addition to vast beds of submerged aquatic vegetation (Potamogeton spp., Vallisneria americana) (Sedgwick and Kroll 2010).

\section{Bird Surveys}

Summer bird surveys have been conducted 4 times in the history of WPMC. J. Bonsall Porter and Dr. Frank W. Langdon surveyed the summer birds of WPMC during the first week of July 1880 (Langdon 1880). John B. Semple and Bayard H. Christy conducted a similar survey 1 July to 3 July 1930 (Christy 1931), and in 1960 John M. Anderson and 5 other observers surveyed the summer resident birds from 2 July to 5 July (Anderson 1960). Finally, in 2017, three WPMC staff members completed survey work on 3 July to 6 July. Observers recorded both visual and auditory detections of birds and estimated abundance to the best of their ability. The entire property was surveyed in all 4 instances using a combination of walking and boats, as well as all-terrain vehicles during the 2017 survey.

In 2017, observers used line transect sampling techniques-following the pre-existing levee system - to survey the property, and approximately 32 kilometers of transect were surveyed. Observers stopped every 200 meters on each transect to record bird observations and search for smaller and more cryptic species. Observers also sampled interior wetland habitat and interior forested habitat ( $<10$ points for both habitat types) using point sampling techniques to detect any species that might be present on the property, but not well represented by levee-based sampling. Two observers worked together for most surveys in 2017, and no transects were sampled more than once to minimize double counting of individuals.

The survey efforts were undoubtedly inconsistent within and between surveys, with no formally established protocol and with varying numbers of observers and observer skill levels. Observers did record count data during all 4 surveys, but due to the inconsistencies in effort this project focused only on presence or absence of different bird species. Included are general interest notes on relative abundance. Langdon (1880) and Christy (1931) also included bird species with a confirmed presence during the summer months at Winous that were not detected within the brief survey window. Similarly, bird species in the 2017 survey that were present outside of the actual survey window, but confirmed present from known observations, were included. Jaccard's coefficients $\left(\mathrm{J}_{s}\right)$ were calculated to compare similarity in species composition among the 4 survey periods after the 2017 survey was completed (Jackson et al. 1989).

\section{RESULTS}

WPMC staff observed 88 bird species during the 2017 survey, similar to the 82 species observed by Anderson (1960) and significantly more than the 71 observed by Christy (1931) and 68 observed by Langdon (1880). Furthermore, WPMC staff documented the presence of 7 additional species outside of the survey window (survey window being the first week in July) but still during June and July: American Avocet (Recurvirostra americana), American Wigeon (Anas americana), Canvasback (Aythya valisineria), Cattle Egret (Bubulcus ibis), Black-necked Stilt (Himantopus mexicanus), Lesser Scaup (Aythya affinis), and Peregrine Falcon (Falco peregrinus) (Table). Thirty-three species were documented on all 4 surveys and 41 species were unique to only a single survey (Table). Eighteen species were recorded during the 2017 survey that had never been recorded during early surveys, but of these it is likely only some-such as Mute Swan (Cygnus olor), Osprey (Pandion haliaetus), Sandhill Crane (Antigone canadensis), and Trumpeter Swan (Cygnus buccinator) —are new colonizers.

Other species documented only in 2017 may or may not have had a historical breeding presence in northwest Ohio. For example, though common now, Herring Gull (Larus argentatus) was never noted before the 2017 survey; even though the species was documented in Ohio dating back to the early 1900s, with breeding evidence after 1960 (Rodewald et al. 2016). Some other species, such as Blue Jay (Cyanocitta cristata) and Black-capped Chickadee (Poecile atricapillus), may simply have been overlooked during earlier surveys as they have a well-documented historical breeding presence in northwest Ohio (Rodewald et al. 2016). 
When comparing species composition among surveys, the greatest difference was between the 2017 and 1880 surveys $\left(\mathrm{J}_{s}=0.514\right)$. Species composition was most similar between the 1930 and 1960 surveys $\left(\mathrm{J}_{s}=0.757\right)$. Of the remaining comparisons, the 1930 and 1880 surveys were the most similar $\left(\mathrm{J}_{s}=0.639\right)$ followed by the 2017 and 1960 surveys $\left(\mathrm{J}_{s}=0.603\right)$, 2017 and 1930 surveys $\left(J_{s}=0.581\right)$, and finally the 1960 and 1880 surveys $\left(\mathrm{J}_{s}=0.544\right)$.

\section{DISCUSSION}

The assumed extirpation of many of the species documented only during the 1880 survey could be attributed to the deforestation and draining of the Great Black Swamp. Extensive European settlement of the region began in the 1840s and Campbell et al. (1995) noted that by 1875 logging and burning had entirely cleared the Great Black Swamp. Blue-gray Gnatcatcher (Polioptila caerulea), Solitary Sandpiper (Tringa solitaria), and Northern Waterthrush (Parkesia noveboracensis) are all inhabitants of wooded wetlands and likely disappeared along with the trees. Similarly, Least Flycatchers (Empidonax minimus), Ruffed Grouse (Bonasa umbellus), Wild Turkey (Meleagris gallopavo), and Wood Thrushes (Hylocichla mustelina) are primarily deciduous and mixed deciduous-coniferous forest obligates. They would have been present in some of the drier sections of the Great Black Swamp before they also disappeared (Anders et al. 1998; Rodewald et al. 2016). The Common Loon (Gavia immer) was the final species that was never observed after the 1880 survey, although their disappearance from WPMC in the summer months was likely linked to reduced water clarity and increased human disturbance at the end of the 19th century (Vermeer 1973; Campbell et al. 1995; Thompson and Price 2006).

After the 1880 survey, the landscape surrounding WPMC remained relatively unchanged for the next several decades as the Great Black Swamp was replaced by small, family-owned farms (Campbell et al. 1995). These small farms were characterized by a diverse array of relatively low-intensity agricultural practices including pasturelands, haylands, and hedgerows (Rollins and Carrol 2001). Relatively large amounts of early successional habitat remained in areas that were unprofitable or infeasible to farm (Campbell et al. 1995; Rollins and Carrol 2001).

Gradually, as the 20th century progressed, lowintensity agricultural techniques and small family farms were consolidated into larger commercial farming operations. This resulted in high-intensity farming, a highly fragmented landscape, and a dramatic reduction in early successional habitat surrounding WPMC (Rollins and Carrol 2001). These changes appear to be reflected in differences between the 1930 and 1960 surveys. For example, Bobolink (Dolichonyx oryzivorus), Eastern Meadowlark (Sturnella magna), and Northern Bobwhite (Colinus virginianus) — all designated as occurring frequently in the 1930 survey-were gone by the 1960 survey. Furthermore, Savannah Sparrow (Passerculussandwichensis) and Ring-necked Pheasant (Phasianus colchicus), along with breeding waterfowl species such as Northern Shoveler (Anas clypeata), Blue-winged Teal (Anas discors), Northern Pintail (Anas acuta), and American Black Duck (Anas rubripes), were all documented during the 1960 survey but extirpated by the 2017 survey. The aforementioned species typically breed in grassland habitat (Heckert 1995; Brennan and Kuvlesky 2005; Rodewald et al. 2016).

During this same period of surveys, the Black Tern (Chlidonias niger), which through the 1960 survey was designated as a common summer breeder at WPMC, also disappeared by 2017 . Black Terns typically form loose nesting colonies comprised of floating nests in large stands of bulrush (Maxson et al. 2007; Rodewald et al. 2016). The extirpation of the Black Tern from WPMC property is likely a result of a substantial decrease in the abundance of bulrush and other emergent vegetation that was once prominent in the region. Several factors (such as introduction of common carp (Cyprinus carpio) and channelization of the Sandusky River) contributed to decreased water quality and increased turbidity, which in turn likely furthered the decline and eventual extirpation of nesting Black Terns in the region (Sedgwick and Kroll 2010).

Four species of interest were encountered on the 2017 survey, 3 of which have never been documented during previous surveys and 1 that has not been observed since the 1880 survey. Sandhill Crane, Osprey, and Trumpeter Swan-never detected previously-were all observed during the 2017 survey, and breeding attempts have been documented in the region. All 3 species are currently experiencing rapid population growth, and their colonization of WPMC is likely a result of finding suitable habitat in an area that was once on the edge of their historical 
range. In the case of Trumpeter Swans, an active introduction program in Ohio also contributed to their population growth (ODNR 2018). In addition, American White Pelicans (Pelecanus erythrorhynchos), not documented since the 1880 survey, have also been growing in abundance. For the last 3 years $(2016$ to 2018) up to 50 non-breeding summer residents have been observed at WPMC.

A final species of note is the Bald Eagle (Haliaeetus leucocephalus), even though it was observed on all 4 surveys. The recovery of Bald Eagles in the contiguous 48 states has been well documented, and locally the collapse and recovery of Bald Eagle populations in northwest Ohio was effectively documented by WPMC surveys (Grier 1982). BaldEagles were noted as "very common" during both the 1880 and 1930 survey; however, by the 1960 survey only a single pair of breeding individuals remained on the property: representing 1 of only 4 nests in Ohio. Following intensive restoration efforts and the banning of DDT, 51 individuals were documented during the 2017 survey and at least 6 active nests were noted on WPMC property.

\section{ACKNOWLEDGEMENTS}

We thank our predecessors who assisted in making this work possible, including F. Langdon, J. Porter, J. Semple, B. Christy, and F. Anderson. We thank all volunteers who assisted with making the 2017 survey possible. We thank the trustees of the Winous Point Marsh Conservancy for their continued support of wetland research and conservation. Finally, we thank the 4 anonymous reviewers whose comments greatly improved this manuscript. 
Table

Observer notes on relative abundance of bird species documented at the Winous Point Marsh Conservancy during the summers of 1880,1930, 1960, and 2017. Notes from the 1880, 1930, and 1960 surveys are from Anderson (1960). Capitalization of common names follows standards of the American Ornithologists' Union (1998). An asterisk $\left(^{*}\right)$ denotes species with either a probable or confirmed breeding presence in Sandusky or Ottawa Counties according to The Second Atlas of Breeding Birds in Ohio (Rodewald et al. 2016). For data analytic purposes, a supplemental spreadsheet titled "Winous Point Supplemental Table" is available at http://hdl.handle.net/1811/88563

\begin{tabular}{|c|c|c|}
\hline Species & Date & Notes \\
\hline \multirow{4}{*}{$\begin{array}{l}\text { Acadian Flycatcher }{ }^{*} \\
\text { (Empidonax virescens) }\end{array}$} & 1880 & Not listed \\
\hline & 1930 & Seen in woods at Winous Point, where the trees stood in water. \\
\hline & 1960 & Not found \\
\hline & 2017 & Not found \\
\hline \multirow{4}{*}{$\begin{array}{l}\text { American Avocet } \\
\text { (Recurvirostra americana) }\end{array}$} & 1880 & Not listed \\
\hline & 1930 & Not listed \\
\hline & 1960 & Not listed \\
\hline & 2017 & $\begin{array}{l}\text { Not found, small flock observed in Horseshoe in summers of } 2015 \\
\text { and } 2018 .\end{array}$ \\
\hline \multirow{4}{*}{$\begin{array}{l}\text { American Bittern * } \\
\text { (Botaurus lentiginosus) }\end{array}$} & 1880 & A few specimens only observed. Breeds. \\
\hline & 1930 & Observed frequently \\
\hline & 1960 & One seen, probably nesting in Gardiner Marsh. \\
\hline & 2017 & Not found, rarely heard during late spring marshbird surveys. \\
\hline \multirow{4}{*}{$\begin{array}{l}\text { American Black Duck* } \\
\text { (Anas rubripes) }\end{array}$} & 1880 & Not listed \\
\hline & 1930 & In the course of two days we saw 3 or 4 pairs. \\
\hline & 1960 & $\begin{array}{l}\text { About } 110 \text { seen. Nesting population estimated at } 20 \text { pairs. Breeding } \\
\text { population has increased in last } 5 \text { years. }\end{array}$ \\
\hline & 2017 & $\begin{array}{l}\text { No breeding individuals observed in last } 10 \text { years. Rarely observed } \\
\text { as a molting summer resident. Banded } 1 \text { to } 5 \text { individuals annually } \\
\text { from } 2014 \text { to } 2017 \text {. Typically, adult males. }\end{array}$ \\
\hline \multirow{4}{*}{$\begin{array}{l}\text { American Coot }{ }^{*} \\
(\text { Fulica americana) }\end{array}$} & 1880 & A few only observed ... A nest containing 2 fresh eggs. \\
\hline & 1930 & $\begin{array}{l}\text { Abundant. Well grown young frequently seen in company with } \\
\text { parents. }\end{array}$ \\
\hline & 1960 & $\begin{array}{l}\text { Twenty-one pairs, } 5 \text { nests, } 6 \text { broods. This species has gradually } \\
\text { increased in number of breeders in the last } 5 \text { years. }\end{array}$ \\
\hline & 2017 & $\begin{array}{l}\text { Not found. Usually detected during spring marshbird surveys, likely } \\
\text { late spring migrants. }\end{array}$ \\
\hline \multirow{4}{*}{$\begin{array}{l}\text { American Crow }{ }^{*} \\
\text { (Corvus brachyrhynchos) }\end{array}$} & 1880 & Not listed \\
\hline & 1930 & Common; less abundant, however, than might be expected. \\
\hline & 1960 & Eleven seen \\
\hline & 2017 & Not found, rarely observed. \\
\hline
\end{tabular}




\section{Table (continued)}

\begin{tabular}{|c|c|c|}
\hline Species & Date & Notes \\
\hline \multirow{4}{*}{$\begin{array}{l}\text { American Goldfinch * } \\
\text { (Spinus tristis) }\end{array}$} & 1880 & Not listed \\
\hline & 1930 & Common \\
\hline & 1960 & Thirty-eight seen \\
\hline & 2017 & Thirty seen; common. \\
\hline \multirow{4}{*}{$\begin{array}{l}\text { American Redstart* } \\
\text { (Setophaga ruticilla) }\end{array}$} & 1880 & Not listed \\
\hline & 1930 & One pair in woods adjacent to the south marsh. \\
\hline & 1960 & Seven seen; apparently this species has increased in recent years. \\
\hline & 2017 & Not found \\
\hline \multirow{4}{*}{$\begin{array}{l}\text { American Robin* } \\
\text { (Turdus migratorius) }\end{array}$} & 1880 & Common \\
\hline & 1930 & Common \\
\hline & 1960 & Nine seen; there are more catbirds than robins at Winous Point. \\
\hline & 2017 & Not found in survey but commonly nests around yard. \\
\hline \multirow[t]{4}{*}{$\begin{array}{l}\text { American White Pelican } \\
\text { (Pelecanus erythrorbynchos) }\end{array}$} & 1880 & $\begin{array}{l}\text { One or two instances of the occurrence of this species in summer } \\
\text { are noted. }\end{array}$ \\
\hline & 1930 & Not found \\
\hline & 1960 & Not found. Stray birds occasionally found in the region. \\
\hline & 2017 & $\begin{array}{l}\text { Twenty-three observed in Horseshoe. Have been summer residents } \\
\text { since } 2014 \text { with as many as } 50 \text { observed at a time. }\end{array}$ \\
\hline \multirow{4}{*}{$\begin{array}{l}\text { American Wigeon * } \\
\text { (Anas americana) }\end{array}$} & 1880 & Not listed \\
\hline & 1930 & Not listed \\
\hline & 1960 & $\begin{array}{l}\text { Four seen; no nesting evidence. Occasional summer birds appear to } \\
\text { be non-breeders. }\end{array}$ \\
\hline & 2017 & $\begin{array}{l}\text { Not found; detected occasionally in recent years at summer duck } \\
\text { banding sites but no evidence of breeding. }\end{array}$ \\
\hline \multirow{4}{*}{$\begin{array}{l}\text { American Woodcock* } \\
\text { (Scolopax minor) }\end{array}$} & 1880 & Not listed \\
\hline & 1930 & $\begin{array}{l}\text { One flushed. The steward of the club, Leonard Jones, reported } \\
\text { a nest this spring in woods near the clubhouse. The nest was } \\
\text { afterward destroyed by high water. }\end{array}$ \\
\hline & 1960 & One seen. One brood raised in woods this spring. \\
\hline & 2017 & $\begin{array}{l}\text { Not found in } 2017 \text {. Occasional summer breeder, } 1 \text { nest found near } \\
\text { dog kennels in } 2016 \text {. }\end{array}$ \\
\hline \multirow[t]{4}{*}{$\begin{array}{l}\text { Bald Eagle* } \\
\text { (Haliaeetus leucocephalus) }\end{array}$} & 1880 & $\begin{array}{l}\text { Several specimens observed during our stay, flapping lazily over the } \\
\text { marsh or taking a siesta on some projecting snag. (A nest described.) }\end{array}$ \\
\hline & 1930 & $\begin{array}{l}\text { Very common. To be seen at all hours. Birds both in adult and } \\
\text { immature plumage. Knowing the existence of a nest in one of the } \\
\text { "elm islands", we did not take time to look it up. }\end{array}$ \\
\hline & 1960 & $\begin{array}{l}\text { Pair with } 1 \text { young beside lane in Gardiner Piece. Pair with } 2 \text { young on } \\
\text { Graveyard Island. The species appears to be less common than formerly. }\end{array}$ \\
\hline & 2017 & Fifty-one seen; very common. At least 6 active nests on the property. \\
\hline
\end{tabular}


Table (continued)

\begin{tabular}{|c|c|c|}
\hline Species & Date & Notes \\
\hline \multirow{4}{*}{$\begin{array}{l}\text { Baltimore Oriole * } \\
\text { (Icterus galbula) }\end{array}$} & 1880 & Common \\
\hline & 1930 & $\begin{array}{l}\text { Not common. A family party noted, the young able to fly. Possibly, } \\
\text { since now the birds are all but silent, they are more plentiful than } \\
\text { would seem. }\end{array}$ \\
\hline & 1960 & Five pairs \\
\hline & 2017 & Twelve seen \\
\hline \multirow{4}{*}{$\begin{array}{l}\text { Bank Swallow* } \\
\text { (Riparia riparia) }\end{array}$} & 1880 & A few only observed. \\
\hline & 1930 & Not found \\
\hline & 1960 & Not found; although very abundant annually in July at clubhouse. \\
\hline & 2017 & Seven seen mixed with tree swallows on power lines. \\
\hline \multirow{4}{*}{$\begin{array}{l}\text { Barn Swallow* } \\
\text { (Hirundo rustica) }\end{array}$} & 1880 & Much less common than the tree swallow. First brood on the wing. \\
\hline & 1930 & Abundant; the most abundant swallow. \\
\hline & 1960 & Seven pairs plus about 30 young. \\
\hline & 2017 & Thirty-three seen around the boat house where they nest often. \\
\hline \multirow{4}{*}{$\begin{array}{l}\text { Belted Kingfisher* } \\
\text { (Megaceryle alcyon) }\end{array}$} & 1880 & Common \\
\hline & 1930 & Common \\
\hline & 1960 & One seen \\
\hline & 2017 & $\begin{array}{l}\text { Two seen; spend most of their time around the township ditch. } \\
\text { Fairly common. }\end{array}$ \\
\hline \multirow{4}{*}{$\begin{array}{l}\text { Black-billed Cuckoo * } \\
\text { (Coccyzus erythropthalmus) }\end{array}$} & 1880 & Not listed \\
\hline & 1930 & Not listed \\
\hline & 1960 & Two seen; probably nesting. \\
\hline & 2017 & One seen; uncommon. \\
\hline \multirow{4}{*}{$\begin{array}{l}\text { Black-capped Chickadee * } \\
\text { (Poecile atricapillus) }\end{array}$} & 1880 & Not listed \\
\hline & 1930 & Not listed \\
\hline & 1960 & Not listed \\
\hline & 2017 & Three seen \\
\hline \multirow{4}{*}{$\begin{array}{l}\text { Black-crowned Night- } \\
\text { Heron * } \\
\text { (Nycticorax nycticorax) }\end{array}$} & 1880 & Not listed \\
\hline & 1930 & Frequently seen, even in daytime, though usually about sunset. \\
\hline & 1960 & $\begin{array}{l}\text { Twenty seen; nesting colony on Mink Island abandoned 1948, } \\
\text { established in catalpa grove beside lane. This colony abandoned } \\
1952 \text {, established on Elm Island. This colony abandoned 1959, no } \\
\text { evidence of nesting presently. }\end{array}$ \\
\hline & 2017 & $\begin{array}{l}\text { Nine flushed from cattail in the marsh. Seems to be an established } \\
\text { roosting site on Little Fox Island. }\end{array}$ \\
\hline
\end{tabular}


Table (continued)

\begin{tabular}{|c|c|c|}
\hline Species & Date & Notes \\
\hline \multirow{4}{*}{$\begin{array}{l}\text { Black-necked Stilt* } \\
\text { (Himantopus mexicanus) }\end{array}$} & 1880 & Not listed \\
\hline & 1930 & Not listed \\
\hline & 1960 & Not listed \\
\hline & 2017 & $\begin{array}{l}\text { Not found, } 3 \text { pairs were commonly observed west of the lane and at } \\
\text { the Metzger's property in the summer of } 2015 \text {. }\end{array}$ \\
\hline \multirow[t]{4}{*}{$\begin{array}{l}\text { Black Tern * } \\
\text { (Chlidonias niger) }\end{array}$} & 1880 & $\begin{array}{l}\text { A very common summer resident in the marsh; nesting . . . In no } \\
\text { instance did we succeed in flushing a bird from the eggs, although } \\
\text { they would appear in pairs to the numbers of } 20 \text { or } 30 \text { and hover } \\
\text { about within a few feet of our heads making a great outcry when we } \\
\text { approached their property. At other times the birds were not at all } \\
\text { gregarious, being usually observed foraging singly or in pairs. }\end{array}$ \\
\hline & 1930 & $\begin{array}{l}\text { Common. Possibly less abundant than Dr. Langdon's comment } \\
\text { would suggest. }\end{array}$ \\
\hline & 1960 & $\begin{array}{l}\text { Seventy-two seen, including } 26 \text { known pairs. Nesting colony on } \\
\text { muskrat houses in Kickapoo. } 2 \text { to } 3 \text { eggs per nest. None hatched by } \\
\text { July 4th. I suspect there was a nesting colony present, although not } \\
\text { discovered, in } 1880 \text { and } 1930 \text {. The dark brown eggs blend very well } \\
\text { with a muskrat house. }\end{array}$ \\
\hline & 2017 & $\begin{array}{l}\text { Not found. Last known nest in the } 1980 \text { s in Lattimore. Not } \\
\text { observed at any time of year in the last } 10 \text { years. }\end{array}$ \\
\hline \multirow{4}{*}{$\begin{array}{l}\text { Blue Jay* } \\
\text { (Cyanocitta cristata) }\end{array}$} & 1880 & Not listed \\
\hline & 1930 & Not listed \\
\hline & 1960 & Not listed \\
\hline & 2017 & Sixteen seen; appears to be more common in July than previously. \\
\hline \multirow[t]{4}{*}{$\begin{array}{l}\text { Blue-gray Gnatcatcher* } \\
\text { (Polioptila caerulea) }\end{array}$} & 1880 & $\begin{array}{l}\text { Evidently this species is here much less common than in southern } \\
\text { Ohio, as our only identification of it rests on the note of a single } \\
\text { individual heard. }\end{array}$ \\
\hline & 1930 & Not found \\
\hline & 1960 & Not found; no recent summer records. \\
\hline & 2017 & Not found \\
\hline \multirow{4}{*}{$\begin{array}{l}\text { Blue-winged Teal* } \\
\text { (Anas discors) }\end{array}$} & 1880 & Breeds; eggs taken in June. \\
\hline & 1930 & $\begin{array}{l}\text { Notation regarding the presence of Mallard applies to this species as } \\
\text { well. We saw none and surmised that already the birds might have } \\
\text { lost their flight feathers and might be in hiding. }\end{array}$ \\
\hline & 1960 & $\begin{array}{l}\text { Saw only } 30 \text {, although breeding population was estimated at } 80 \\
\text { pairs. Shows marked increase in nesting in last } 4 \text { years. }\end{array}$ \\
\hline & 2017 & $\begin{array}{l}\text { One nest located in Horseshoe in } 2014 \text {. Rarely observed in } \\
\text { summer. }\end{array}$ \\
\hline
\end{tabular}


Table (continued)

\begin{tabular}{|c|c|c|}
\hline Species & Date & Notes \\
\hline \multirow{4}{*}{$\begin{array}{l}\text { Bobolink* } \\
\text { (Dolichonyx oryzivorus) }\end{array}$} & 1880 & A few specimens only observed. \\
\hline & 1930 & Common in fields at northern base of Winous Point. \\
\hline & 1960 & Not found, although it nests in fields north of the marsh. \\
\hline & 2017 & Not found \\
\hline \multirow{4}{*}{$\begin{array}{l}\text { Bonaparte's Gull } \\
\text { (Chroicocephalus } \\
\text { philadelphia) }\end{array}$} & 1880 & Not listed \\
\hline & 1930 & Not listed \\
\hline & 1960 & Not listed \\
\hline & 2017 & Not found, but abundant on Muddy Creek Bay in summer. \\
\hline \multirow{4}{*}{$\begin{array}{l}\text { Brown Thrasher* } \\
\text { (Toxostoma rufum) }\end{array}$} & 1880 & Rather common \\
\hline & 1930 & Not found \\
\hline & 1960 & One pair \\
\hline & 2017 & One seen \\
\hline \multirow{4}{*}{$\begin{array}{l}\text { Brown-headed Cowbird }{ }^{*} \\
\text { (Molothrus ater) }\end{array}$} & 1880 & Common \\
\hline & 1930 & Common \\
\hline & 1960 & Fifty-nine seen \\
\hline & 2017 & Eight seen; common. \\
\hline \multirow{4}{*}{$\begin{array}{l}\text { Bufflehead } \\
\text { (Bucephala albeola) }\end{array}$} & 1880 & Frequently seen and taken in summer. (Evidently not seen in 1880. .) \\
\hline & 1930 & Not found \\
\hline & 1960 & Not found \\
\hline & 2017 & Not found \\
\hline \multirow[t]{4}{*}{$\begin{array}{l}\text { Canada Goose* } \\
\text { (Branta canadensis) }\end{array}$} & 1880 & $\begin{array}{l}\text { Mr. Porter informs me that individuals of this species are frequently } \\
\text { seen in the marsh in summer, having probably been crippled during } \\
\text { the shooting season and thereby prevented from migrating. }\end{array}$ \\
\hline & 1930 & Not found \\
\hline & 1960 & $\begin{array}{l}\text { Not found. About } 600 \text { now wintering on the marsh; about 1,000 } \\
\text { winter on Gressman farm about } 10 \text { miles west. }\end{array}$ \\
\hline & 2017 & $\begin{array}{l}\text { One hundred ninety-eight observed; the most common breeding } \\
\text { waterfowl species. }\end{array}$ \\
\hline \multirow{4}{*}{$\begin{array}{l}\text { Canvasback } \\
\text { (Aythya valisineria) }\end{array}$} & 1880 & Of occasional occurrence in summer. (Evidently not seen.) \\
\hline & 1930 & Not found \\
\hline & 1960 & Not found \\
\hline & 2017 & Not found \\
\hline
\end{tabular}


Table (continued)

\begin{tabular}{|c|c|c|}
\hline Species & Date & Notes \\
\hline \multirow{4}{*}{$\begin{array}{l}\text { Cattle Egret } \\
\text { (Bubulcus ibis) }\end{array}$} & 1880 & Not Listed \\
\hline & 1930 & Not Listed \\
\hline & 1960 & Not Listed \\
\hline & 2017 & $\begin{array}{l}\text { None observed during survey. Rarely, but occasionally observed in } \\
\text { early summer. }\end{array}$ \\
\hline \multirow{4}{*}{$\begin{array}{l}\text { Cedar Waxwing * } \\
\text { (Bombycilla cedrorum) }\end{array}$} & 1880 & Observed breeding in 1878 . \\
\hline & 1930 & Two pairs noted in different places. \\
\hline & 1960 & Not found, although common in migration. \\
\hline & 2017 & One seen; foraging in a mulberry tree near the Locust Grove. \\
\hline \multirow{4}{*}{$\begin{array}{l}\text { Chestnut-sided Warbler* } \\
\text { (Setophaga pensylvanica) }\end{array}$} & 1880 & Not found \\
\hline & 1930 & Not found \\
\hline & 1960 & One female seen; presumably accidental. \\
\hline & 2017 & Not found \\
\hline \multirow{4}{*}{$\begin{array}{l}\text { Chimney Swift* } \\
\text { (Chaetura pelagica) }\end{array}$} & 1880 & Not listed \\
\hline & 1930 & Abundant \\
\hline & 1960 & Fifteen to 18 pairs estimated. \\
\hline & 2017 & Not found \\
\hline \multirow[t]{4}{*}{$\begin{array}{l}\text { Cliff Swallow * } \\
\text { (Petrochelidon pyrrhonota) }\end{array}$} & 1880 & $\begin{array}{l}\text { Many observed carrying mud; doubles to repair their nests for the } \\
\text { reception of the second brood. }\end{array}$ \\
\hline & 1930 & $\begin{array}{l}\text { Not found. A neighboring farmer, in reply to a question, said } \\
\text { that the swallows used to nest under the eaves of his barn; that } \\
\text { the sparrows drove them away and for a time there were few or } \\
\text { none; and that now, nesting within the barn, they had increased in } \\
\text { numbers. The farmer's confusion of two species needs but a word: } \\
\text { the earlier birds were Cliff Swallows, those of the later years, Barn } \\
\text { Swallows. }\end{array}$ \\
\hline & 1960 & $\begin{array}{l}\text { Not found. There was a small colony on neighbor's barn in } 1947 \text {; } \\
\text { lasted } 1 \text { year. }\end{array}$ \\
\hline & 2017 & Not found \\
\hline \multirow[t]{4}{*}{$\begin{array}{l}\text { Common Gallinule * } \\
\text { (Gallinula galeata) }\end{array}$} & 1880 & $\begin{array}{l}\text { A very common species, breeding abundantly in the more open } \\
\text { portions of the marsh. (Eggs taken and young observed.) }\end{array}$ \\
\hline & 1930 & $\begin{array}{l}\text { Very common. The most abundant aquatic bird. Empty nests found } \\
\text { and half-grown young frequently seen. }\end{array}$ \\
\hline & 1960 & Sixty-one pairs seen, 11 nests, 15 broods. \\
\hline & 2017 & $\begin{array}{l}\text { Five seen or heard, common breeder, nests occasionally } \\
\text { encountered. }\end{array}$ \\
\hline
\end{tabular}


Table (continued)

\begin{tabular}{|c|c|c|}
\hline Species & Date & Notes \\
\hline \multirow{4}{*}{$\begin{array}{l}\text { Common Grackle* } \\
\text { (Quiscalus quiscula) }\end{array}$} & 1880 & Several shot for identification. \\
\hline & 1930 & Very common \\
\hline & 1960 & One hundred and twelve seen. \\
\hline & 2017 & Twenty-seven seen; common. \\
\hline \multirow{4}{*}{$\begin{array}{l}\text { Common Loon } \\
\text { (Gavia immer) }\end{array}$} & 1880 & Three or four specimens observed in summer. \\
\hline & 1930 & Not found \\
\hline & 1960 & Not found; no recent summer records. \\
\hline & 2017 & Not found; observed occasionally during spring migration. \\
\hline \multirow[t]{4}{*}{$\begin{array}{l}\text { Common Tern * } \\
\text { (Sterna hirundo) }\end{array}$} & 1880 & $\begin{array}{l}\text { Four specimens taken and others observed by Mr. Porter. We were } \\
\text { informed that the "larger" terns (probably this species) are quite } \\
\text { plentiful in the marsh during rough weather, which drives them in } \\
\text { from the lake. }\end{array}$ \\
\hline & 1930 & $\begin{array}{l}\text { Noted twice or three times, singly or in pairs, flying over bay or } \\
\text { marsh; and, on another occasion, in late afternoon, a compact } \\
\text { company of } 30 \text { to } 40 \text {, flying rather high, up the bay and up the } \\
\text { wind, toward an approaching shower. }\end{array}$ \\
\hline & 1960 & Two seen \\
\hline & 2017 & Four seen on the bay. Known to nest at 2 locations locally. \\
\hline \multirow[t]{4}{*}{$\begin{array}{l}\text { Common Yellowthroat* } \\
\text { (Geothlypis trichas) }\end{array}$} & 1880 & $\begin{array}{l}\text { Rather common, though not so abundant as would be expected, } \\
\text { considering the adaptation of the locality to its habits. }\end{array}$ \\
\hline & 1930 & Abundant \\
\hline & 1960 & Twenty-five seen \\
\hline & 2017 & Twenty-two seen \\
\hline \multirow{4}{*}{$\begin{array}{l}\text { Cooper's Hawk }{ }^{*} \\
\text { (Accipiter cooperii) }\end{array}$} & 1880 & May 1877 \\
\hline & 1930 & Not found \\
\hline & 1960 & Not found \\
\hline & 2017 & Not observed but known to be common summer resident. \\
\hline \multirow{4}{*}{$\begin{array}{l}\text { Dickcissel * } \\
(\text { Spiza americana })\end{array}$} & 1880 & Three or four species observed and taken. \\
\hline & 1930 & $\begin{array}{l}\text { Not found on this visit. Three years ago (1927) a colony was found } \\
\text { in a clover field within a mile to the northward from Winous Point. } \\
\text { This year (1930) the clover crop was poor and the fields few. A } \\
\text { hasty survey of fields of clover and of alfalfa passed on the way from } \\
\text { Winous Point to Port Clinton failed to disclose the bird. }\end{array}$ \\
\hline & 1960 & $\begin{array}{l}\text { Not found. Throughout Ottawa County numbers of Dickcissels } \\
\text { fluctuate widely from year to year, but they have not been common } \\
\text { adjacent to Winous Point for over } 10 \text { years. }\end{array}$ \\
\hline & 2017 & $\begin{array}{l}\text { Twelve seen; seem to be confined to the northern edge of the } \\
\text { property near Latimore and Gardiner. }\end{array}$ \\
\hline
\end{tabular}


Table (continued)

\begin{tabular}{|c|c|c|}
\hline Species & Date & Notes \\
\hline \multirow{4}{*}{$\begin{array}{l}\text { Double-crested } \\
\text { Cormorant }{ }^{*} \\
\text { (Phalacrocorax auritus) }\end{array}$} & 1880 & Two specimens, male and female, taken in June 1878. \\
\hline & 1930 & Not found \\
\hline & 1960 & Not found \\
\hline & 2017 & Only 2 observed; frequent summer resident, more common in fall. \\
\hline \multirow{4}{*}{$\begin{array}{l}\text { Downy Woodpecker* } \\
\text { (Dryobates pubescens) }\end{array}$} & 1880 & Not listed \\
\hline & 1930 & Common in woods adjacent to the marsh. \\
\hline & 1960 & Two pairs seen. \\
\hline & 2017 & Twenty-three seen; most common woodpecker species seen in summer. \\
\hline \multirow{4}{*}{$\begin{array}{l}\text { Eastern Bluebird * } \\
\text { (Sialia sialis) }\end{array}$} & 1880 & Not listed \\
\hline & 1930 & Rare. Noted at Port Clinton. \\
\hline & 1960 & Not found \\
\hline & 2017 & Not found. Occasionally observed nearby Hwy. 53. \\
\hline \multirow[t]{4}{*}{$\begin{array}{l}\text { Eastern Kingbird* } \\
\text { (Tyrannus tyrannus) }\end{array}$} & 1880 & $\begin{array}{l}\text { In the woods and groves bordering the marsh we met with this } \\
\text { species in greater number than I have ever seen it elsewhere; they } \\
\text { were not in flocks, but in some places almost every tree appeared } \\
\text { to be occupied by a pair of these vociferous insect collectors. The } \\
\text { abundance of the deer fly and other insect pests about the marsh } \\
\text { at this season is such that the kingbird is an exceedingly welcome } \\
\text { addition to the fauna. }\end{array}$ \\
\hline & 1930 & $\begin{array}{l}\text { Abundant. Dr. Langdon's words, if applied to the present status, are } \\
\text { not inappropriate; perhaps "almost every tree" would be thought } \\
\text { hyperbole, but Dr. Langdon suggests as much. }\end{array}$ \\
\hline & 1960 & Twelve seen; common nester, one nest seen on census. \\
\hline & 2017 & Twenty-four seen; common summer bird. \\
\hline \multirow{4}{*}{$\begin{array}{l}\text { Eastern Meadowlark* } \\
\text { (Sturnella magna) }\end{array}$} & 1880 & Common \\
\hline & 1930 & Common \\
\hline & 1960 & Not found, although it is a common permanent resident. \\
\hline & 2017 & Not found. \\
\hline \multirow[t]{4}{*}{$\begin{array}{l}\text { Eastern Phoebe * } \\
\text { (Sayornis phoebe) }\end{array}$} & 1880 & $\begin{array}{l}\text { In spite of the abundance of insect food, we observed but a few of } \\
\text { this species. }\end{array}$ \\
\hline & 1930 & One noted \\
\hline & 1960 & Not found; has nested on foot bridge in recent years. \\
\hline & 2017 & Three seen; a pair nesting under the overhang of the bungalow. \\
\hline \multirow[t]{4}{*}{$\begin{array}{l}\text { Eastern Screech-Owl * } \\
\text { (Megascops asio) }\end{array}$} & 1880 & $\begin{array}{l}\text { Two specimens, young of the year, taken; both were in the grey } \\
\text { phase of plumage. }\end{array}$ \\
\hline & 1930 & One seen, in red phase. \\
\hline & 1960 & $\begin{array}{l}\text { Two seen; nest frequently in wood duck boxes and hollow trees. Probably } \\
\text { about } 10 \text { pairs on club lane. Several young banded annually. }\end{array}$ \\
\hline & 2017 & One pair seen. \\
\hline
\end{tabular}


Table (continued)

\begin{tabular}{|c|c|c|}
\hline Species & Date & Notes \\
\hline \multirow{4}{*}{$\begin{array}{l}\text { Eastern Wood-Pewee * } \\
\text { (Contopus virens) }\end{array}$} & 1880 & Common \\
\hline & 1930 & Common \\
\hline & 1960 & Two seen \\
\hline & 2017 & Four seen \\
\hline \multirow{4}{*}{$\begin{array}{l}\text { European Starling * } \\
\text { (Sturnus vulgaris) }\end{array}$} & 1880 & Not listed \\
\hline & 1930 & Abundant; already gathered in flocks. \\
\hline & 1960 & $\begin{array}{l}\text { Two hundred estimated in small flocks of } 10 \text { to } 50 \text {. One pair tried } \\
\text { to nest in side of the eagless nest. }\end{array}$ \\
\hline & 2017 & $\begin{array}{l}\text { Seventy-three seen; commonly seen foraging in the lawn around } \\
\text { manager's house and bungalow. }\end{array}$ \\
\hline \multirow{4}{*}{$\begin{array}{l}\text { Field Sparrow* } \\
\text { (Spizella pusilla) }\end{array}$} & 1880 & Summer resident \\
\hline & 1930 & A few noted on a farm adjacent to the south marsh. \\
\hline & 1960 & Not found \\
\hline & 2017 & One heard in the North Bay property. \\
\hline \multirow{4}{*}{$\begin{array}{l}\text { Gray Catbird * } \\
\text { (Dumetella carolinensis) }\end{array}$} & 1880 & Not listed \\
\hline & 1930 & Common; present in suitable localities. \\
\hline & 1960 & Eleven seen \\
\hline & 2017 & Forty-one seen \\
\hline \multirow{4}{*}{$\begin{array}{l}\text { Great Blue Heron * } \\
\text { (Ardea herodias) }\end{array}$} & 1880 & Very common and shy, as usual. \\
\hline & 1930 & Very common \\
\hline & 1960 & Rookery on Elm Island contains over 590 nests. \\
\hline & 2017 & $\begin{array}{l}\text { Forty observed; common across the property. The rookery mentioned } \\
\text { on Elm Island has since been abandoned, as was a previous rookery } \\
\text { in Mackey woods. Rookery exists on Ottawa Shooting Club. }\end{array}$ \\
\hline \multirow{4}{*}{$\begin{array}{l}\text { Great Crested Flycatcher* } \\
\text { (Myiarchus crinitus) }\end{array}$} & 1880 & Not listed \\
\hline & 1930 & $\begin{array}{l}\text { A pair, repeatedly noted in coming and going through the woods on } \\
\text { Winous Point, gave the impression, perhaps subject to correction, } \\
\text { that the species is a common one. }\end{array}$ \\
\hline & 1960 & Three seen; probably nesting. \\
\hline & 2017 & Six seen; uncommon. \\
\hline \multirow{4}{*}{$\begin{array}{l}\text { Great Egret* } \\
(\text { Ardea alba })\end{array}$} & 1880 & Usually common in August and September. \\
\hline & 1930 & $\begin{array}{l}\text { Mr. Semple in the late summer of } 1929 \text { observed a flock of } 12 \text {, } \\
\text { which roosted in woods near the clubhouse. }\end{array}$ \\
\hline & 1960 & $\begin{array}{l}\text { Five seen; one nesting pair, prior to } 1959 \text {, about } 25 \text { pairs nested on } \\
\text { Elm Island, the only breeding colony on the mainland of Ohio. }\end{array}$ \\
\hline & 2017 & Ninety observed; very common but no rookery on the property. \\
\hline
\end{tabular}


Table (continued)

\begin{tabular}{|c|c|c|}
\hline Species & Date & Notes \\
\hline \multirow{4}{*}{$\begin{array}{l}\text { Great Horned Owl* } \\
\text { (Bubo virginianus) }\end{array}$} & 1880 & Not listed \\
\hline & 1930 & Not listed \\
\hline & 1960 & $\begin{array}{l}\text { One pair plus single bird seen. Nests in woods above clubhouse, } \\
\text { occupied an eagle nest this year. Probably at least three pairs nesting } \\
\text { on club grounds. An adult was collected at the club in the spring of } \\
\text { 1881. Probably was overlooked on previous censuses. }\end{array}$ \\
\hline & 2017 & One seen flying in woods east of Gottrons. Common summer resident. \\
\hline \multirow{4}{*}{$\begin{array}{l}\text { Greater Yellowlegs } \\
\text { (Tringa melanoleuca) }\end{array}$} & 1880 & July 1879 \\
\hline & 1930 & Not found \\
\hline & 1960 & Not found \\
\hline & 2017 & $\begin{array}{l}\text { Not found. Only } 26 \text { banded during summer months from } 2006 \text { to } \\
2015 \text {. }\end{array}$ \\
\hline \multirow{4}{*}{$\begin{array}{l}\text { Green Heron* } \\
\text { (Butorides virescens) }\end{array}$} & 1880 & Breeds \\
\hline & 1930 & Common, nest found. \\
\hline & 1960 & Three nesting pairs seen. \\
\hline & 2017 & $\begin{array}{l}\text { Not found during the survey but frequently encountered } \\
\text { during summer. }\end{array}$ \\
\hline \multirow{4}{*}{$\begin{array}{l}\text { Green-winged Teal * } \\
\text { (Anas crecca) }\end{array}$} & 1880 & Not listed \\
\hline & 1930 & Not listed \\
\hline & 1960 & $\begin{array}{l}\text { Two seen. First nest for Winous Point discovered in Lattimore } \\
\text { Marsh June 16th. I collected downy young on Ohio side of } \\
\text { Pymatuning Lake in } 1937 \text {. Aside from the nest in the Lattimore, I } \\
\text { know of but one other nest for this species in Ohio. }\end{array}$ \\
\hline & 2017 & $\begin{array}{l}\text { Rare nester locally, none known on Winous since } 2008 \text {. Observed } \\
\text { most summers but in very low numbers, likely non-breeders. }\end{array}$ \\
\hline \multirow{4}{*}{$\begin{array}{l}\text { Hairy Woodpecker }{ }^{*} \\
\text { (Dryobates villosus) }\end{array}$} & 1880 & Not listed \\
\hline & 1930 & Not listed \\
\hline & 1960 & Not listed \\
\hline & 2017 & $\begin{array}{l}\text { Two seen; may have been confused with Downy Woodpeckers on } \\
\text { previous surveys. }\end{array}$ \\
\hline \multirow{4}{*}{$\begin{array}{l}\text { Herring Gull * } \\
\text { (Larus argentatus) }\end{array}$} & 1880 & Not listed \\
\hline & 1930 & Not listed \\
\hline & 1960 & Not listed \\
\hline & 2017 & One seen on Muddy Creek Bay. \\
\hline
\end{tabular}


Table (continued)

\begin{tabular}{|c|c|c|}
\hline Species & Date & Notes \\
\hline \multirow{4}{*}{$\begin{array}{l}\text { Hooded Merganser* } \\
\text { (Lophodytes cucullatus) }\end{array}$} & 1880 & Not listed \\
\hline & 1930 & Not listed \\
\hline & 1960 & Not listed \\
\hline & 2017 & Common summer breeder in marsh, broods frequently observed. \\
\hline \multirow{4}{*}{$\begin{array}{l}\text { House Finch * } \\
\text { (Haemorhous mexicanus) }\end{array}$} & 1880 & Not listed \\
\hline & 1930 & Not listed \\
\hline & 1960 & Not listed \\
\hline & 2017 & Three seen in trees between Darr and Bunker Hill. \\
\hline \multirow{4}{*}{$\begin{array}{l}\text { House Sparrow* } \\
\text { (Passer domesticus) }\end{array}$} & 1880 & Common at Port Clinton, within 5 or 6 miles of the marsh. \\
\hline & 1930 & Abundant about the club buildings and on adjacent farms. \\
\hline & 1960 & $\begin{array}{l}\text { Twelve pairs about the buildings. Two pairs trying unsuccessfully to } \\
\text { nest in side of eagle's nest. }\end{array}$ \\
\hline & 2017 & $\begin{array}{l}\text { Five seen; didn't spend much time on this species. Common around } \\
\text { the buildings. }\end{array}$ \\
\hline \multirow{4}{*}{$\begin{array}{l}\text { House Wren * } \\
\text { (Troglodytes aedon) }\end{array}$} & 1880 & Breeds \\
\hline & 1930 & Abundant in all suitable localities. \\
\hline & 1960 & Twenty seen; including 7 males, 5 juveniles, and 8 undetermined. \\
\hline & 2017 & Nineteen seen and heard in Mackey Woods. \\
\hline \multirow{4}{*}{$\begin{array}{l}\text { Indigo Bunting * } \\
\text { (Passerina cyanea) }\end{array}$} & 1880 & Very common \\
\hline & 1930 & $\begin{array}{l}\text { Certainly common and well distributed. I should be disposed to } \\
\text { drop the "very" from Dr. Langdon's comment. }\end{array}$ \\
\hline & 1960 & Twenty-five seen; status probably about the same as in 1930 . \\
\hline & 2017 & Forty-two seen; common species. \\
\hline \multirow{4}{*}{$\begin{array}{l}\text { Killdeer }{ }^{*} \\
\text { (Charadrius vociferus) }\end{array}$} & 1880 & Breeds \\
\hline & 1930 & Several noted, among them birds of the year. \\
\hline & 1960 & $\begin{array}{l}\text { Only } 3 \text { seen during this period; much more abundant during } \\
\text { nesting season. }\end{array}$ \\
\hline & 2017 & Six seen; common. \\
\hline \multirow{4}{*}{$\begin{array}{l}\text { King Rail * } \\
\text { (Rallus elegans) }\end{array}$} & 1880 & One specimen taken by Mr. Porter July 3rd. \\
\hline & 1930 & Not found \\
\hline & 1960 & $\begin{array}{l}\text { One seen; this game bird was abundant on the marsh in the } \\
\text { late } 1940 \text { s, reached its peak in 1952, and has been declining in } \\
\text { numbers since. }\end{array}$ \\
\hline & 2017 & $\begin{array}{l}\text { Six seen; one adult with } 5 \text { young crossing the dike north of North } \\
\text { Lily. Occasionally other broods and individuals have been seen } \\
\text { since } 2008 \text {. We have banded } 18 \text { known summer resident birds } \\
\text { since } 2014 \text {. }\end{array}$ \\
\hline
\end{tabular}


Table (continued)

\begin{tabular}{|c|c|c|}
\hline Species & Date & Notes \\
\hline \multirow{4}{*}{$\begin{array}{l}\text { Least Bittern * } \\
\text { (Ixobrychus exilis) }\end{array}$} & 1880 & Quite common (eggs taken). \\
\hline & 1930 & Quite common \\
\hline & 1960 & Ten seen; nests in dense emergent vegetation. \\
\hline & 2017 & $\begin{array}{l}\text { One seen; commonly flushed in dense emergent vegetation in April } \\
\text { and May and is a known breeder. }\end{array}$ \\
\hline \multirow{4}{*}{$\begin{array}{l}\text { Least Flycatcher }{ }^{*} \\
\text { (Empidonax minimus) }\end{array}$} & 1880 & One specimen taken. \\
\hline & 1930 & Not found \\
\hline & 1960 & Not found; no recent summer records. \\
\hline & 2017 & Not found \\
\hline \multirow{4}{*}{$\begin{array}{l}\text { Least Sandpiper } \\
\text { (Calidris minutilla) }\end{array}$} & 1880 & Not listed \\
\hline & 1930 & Not found \\
\hline & 1960 & Seven seen \\
\hline & 2017 & $\begin{array}{l}\text { Six seen. Over } 3000 \text { banded during summer months from } 2006 \\
\text { to } 2015 \text {. }\end{array}$ \\
\hline \multirow{4}{*}{$\begin{array}{l}\text { Lesser Scaup } \\
\text { (Aythya affinis) }\end{array}$} & 1880 & Not listed \\
\hline & 1930 & Not listed \\
\hline & 1960 & One hen seen, apparently non-breeding bird. \\
\hline & 2017 & $\begin{array}{l}\text { Not found; } 1 \text { individual observed on a trial camera photo at } \\
\text { summer duck banding site in } 2017 \text {. }\end{array}$ \\
\hline \multirow{4}{*}{$\begin{array}{l}\text { Lesser Yellowlegs } \\
\text { (Tringa flavipes) }\end{array}$} & 1880 & Not listed \\
\hline & 1930 & $\begin{array}{l}\text { A party of four jumped from marsh, and by whistling called back, } \\
\text { within easy range of identification. }\end{array}$ \\
\hline & 1960 & Four seen \\
\hline & 2017 & $\begin{array}{l}\text { Seventy-three seen; mostly in the southeast corner of Horseshoe. } \\
\text { Banded } 662 \text { during summer months from } 2006 \text { to } 2015 \text {. }\end{array}$ \\
\hline \multirow{4}{*}{$\begin{array}{l}\text { Loggerhead Shrike } \\
\text { (Lanius ludovicianus) }\end{array}$} & 1880 & A single pair observed. \\
\hline & 1930 & A single bird seen by the highway near Sandusky. \\
\hline & 1960 & Two pairs seen. \\
\hline & 2017 & Not found \\
\hline \multirow{4}{*}{$\begin{array}{l}\text { Mallard * } \\
\text { (Anas platyrhynchos) }\end{array}$} & 1880 & Breeds. (Evidently none were observed.) \\
\hline & 1930 & $\begin{array}{l}\text { Generally recognized to be a nesting species. The steward, well } \\
\text { acquainted with waterfowl, reported an individual feeding with his } \\
\text { live decoys at the edge of the marsh. (Evidently none were seen.) }\end{array}$ \\
\hline & 1960 & $\begin{array}{l}\text { About } 200 \text { seen; nesting population estimated at } 110 \text { pairs. Three } \\
\text { broods seen. One hundred molting drakes estimated on marsh. } \\
\text { Shows marked increase in last } 5 \text { years. }\end{array}$ \\
\hline & 2017 & $\begin{array}{l}\text { Common local breeder and molts by the thousands in late } \\
\text { summer. We have banded approximately } 1,500 \text { in the } \\
\text { summertime since } 2015 \text {. }\end{array}$ \\
\hline
\end{tabular}


Table (continued)

\begin{tabular}{|c|c|c|}
\hline Species & Date & Notes \\
\hline \multirow[t]{4}{*}{$\begin{array}{l}\text { Marsh Wren * } \\
\text { (Cistothorus palustris) }\end{array}$} & 1880 & $\begin{array}{l}\text { An abundant species, its lively grasshopper-like notes resounding } \\
\text { on all sides in the marsh. Five eggs appears to constitute a full set, } \\
\text { although we took sets of four that were slightly incubated; and } \\
\text { while most of those taken were fresh, or nearly so, a few sets were } \\
\text { far advanced in incubation. (July } 2 \text { nd.) }\end{array}$ \\
\hline & 1930 & Would accept Dr. Langdon's statement of abundance. \\
\hline & 1960 & Thirty-five pairs seen, estimated about 100 pairs on entire marsh. \\
\hline & 2017 & $\begin{array}{l}\text { Nest in extremely high densities and closely linked to cattail stands. } \\
\text { Hundreds of breeding individuals. }\end{array}$ \\
\hline \multirow{4}{*}{$\begin{array}{l}\text { Mourning Dove * } \\
\text { (Zenaida macroura) }\end{array}$} & 1880 & Nest containing half fledged young observed July 2nd. \\
\hline & 1930 & Very common. Many young flown from nests. \\
\hline & 1960 & $\begin{array}{l}\text { At least } 50 \text { pairs. Many fledglings have been banded annually in last } \\
10 \text { years. }\end{array}$ \\
\hline & 2017 & Eleven seen; common. \\
\hline \multirow{4}{*}{$\begin{array}{l}\text { Mute Swan * } \\
\text { (Cygnus olor) }\end{array}$} & 1880 & Not listed \\
\hline & 1930 & Not listed \\
\hline & 1960 & Not listed \\
\hline & 2017 & Present locally but uncommon specifically at Winous Point. \\
\hline \multirow{4}{*}{$\begin{array}{l}\text { Northern Bobwhite }{ }^{*} \\
\text { (Colinus virginianus) }\end{array}$} & 1880 & Not listed \\
\hline & 1930 & Frequently heard whistling on grassy upland adjacent to the marsh. \\
\hline & 1960 & $\begin{array}{l}\text { Not found. No records for the marsh or adjacent fields for at } \\
\text { least } 13 \text { years. }\end{array}$ \\
\hline & 2017 & Not found. No records since 1940s. \\
\hline \multirow{4}{*}{$\begin{array}{l}\text { Northern Cardinal * } \\
\text { (Cardinalis cardinalis) }\end{array}$} & 1880 & Rare \\
\hline & 1930 & Common \\
\hline & 1960 & Common. Three observed. \\
\hline & 2017 & Common. Thirty-four observed. \\
\hline \multirow{4}{*}{$\begin{array}{l}\text { Northern Flicker * } \\
\text { (Colaptes auratus) }\end{array}$} & 1880 & Not listed \\
\hline & 1930 & Common \\
\hline & 1960 & Twelve seen, including nesting pair in yard. \\
\hline & 2017 & Fourteen seen \\
\hline \multirow{4}{*}{$\begin{array}{l}\text { Northern Mockingbird * } \\
\text { (Mimus polyglottos) }\end{array}$} & 1880 & Not listed \\
\hline & 1930 & Not listed \\
\hline & 1960 & Not listed \\
\hline & 2017 & One seen in landscaping around the bungalow. \\
\hline
\end{tabular}


Table (continued)

\begin{tabular}{|c|c|c|}
\hline Species & Date & Notes \\
\hline \multirow{4}{*}{$\begin{array}{l}\text { Northern Pintail * } \\
\text { (Anas acuta) }\end{array}$} & 1880 & Not listed \\
\hline & 1930 & Not listed \\
\hline & 1960 & $\begin{array}{l}\text { About } 100 \text { molting birds west of Horseshoe Island. Breeding } \\
\text { population estimated at } 60 \text { pairs. In recent years has become an } \\
\text { uncommon but regular nester. }\end{array}$ \\
\hline & 2017 & Not known to nest or molt locally since 2008. \\
\hline \multirow{4}{*}{$\begin{array}{l}\text { Northern Rough-winged } \\
\text { Swallow * } \\
\text { (Stelgidopteryx serripennis) }\end{array}$} & 1880 & Not listed \\
\hline & 1930 & Common \\
\hline & 1960 & Not found; no recent summer records. \\
\hline & 2017 & Ten seen near Fox Island bridge on Green Creek. \\
\hline \multirow{4}{*}{$\begin{array}{l}\text { Northern Shoveler * } \\
\text { (Anas clypeata) }\end{array}$} & 1880 & Not listed \\
\hline & 1930 & Not listed \\
\hline & 1960 & $\begin{array}{l}\text { Fifteen seen, including } 3 \text { pairs and } 9 \text { males. At least } 2 \text { males } \\
\text { defended nesting territory in the research area this spring. Has } \\
\text { definitely become established as a nesting species in recent years. }\end{array}$ \\
\hline & 2017 & Not observed as a summer resident. \\
\hline \multirow[t]{4}{*}{$\begin{array}{l}\text { Northern Waterthrush } \\
\text { (Parkesia noveboracensis) }\end{array}$} & 1880 & $\begin{array}{l}\text { One specimen only observed; so close that there could be no } \\
\text { question as to its identity. }\end{array}$ \\
\hline & 1930 & Not found \\
\hline & 1960 & Not found \\
\hline & 2017 & Not found \\
\hline \multirow{4}{*}{$\begin{array}{l}\text { Orchard Oriole * } \\
\text { (Icterus spurius) }\end{array}$} & 1880 & Common \\
\hline & 1930 & $\begin{array}{l}\text { One pair noted, nesting near clubhouse; a second pair along the } \\
\text { highway two miles away. Manifestly not a common species. }\end{array}$ \\
\hline & 1960 & Two pairs; not considered common. \\
\hline & 2017 & Twelve seen \\
\hline \multirow{4}{*}{$\begin{array}{l}\text { Osprey* } \\
\text { (Pandion haliaetus) }\end{array}$} & 1880 & Not listed \\
\hline & 1930 & Not listed \\
\hline & 1960 & Not listed \\
\hline & 2017 & $\begin{array}{l}\text { One seen; individual was perched on cell tower along Lattimore } \\
\text { Road. Osprey platforms erected in } 2014 \text { (Metzger's) and } 2016 \\
\text { (Lattimore). Nest building activity at Lattimore in } 2016 \text { and } \\
\text { 2017. Rare summer resident since 2008. Known to nest at Ottawa } \\
\text { National Wildlife Refuge. }\end{array}$ \\
\hline \multirow{4}{*}{$\begin{array}{l}\text { Pectoral Sandpiper } \\
\text { (Calidris melanotos) }\end{array}$} & 1880 & Not listed \\
\hline & 1930 & Not listed \\
\hline & 1960 & Three seen \\
\hline & 2017 & Not seen but common; 906 banded in summer from 2006 to 2015 . \\
\hline
\end{tabular}


Table (continued)

\begin{tabular}{|c|c|c|}
\hline Species & Date & Notes \\
\hline \multirow{4}{*}{$\begin{array}{l}\text { Peregrine Falcon } \\
\text { (Falco peregrinus) }\end{array}$} & 1880 & Not listed \\
\hline & 1930 & Not listed \\
\hline & 1960 & Not listed \\
\hline & 2017 & $\begin{array}{l}\text { None seen during surveys but known to nest in Port Clinton and } \\
\text { Toledo. Occasionally observed chasing shorebirds in summer and } \\
\text { teal in early fall. One captured in } 2009 \text { during shorebird mist- } \\
\text { netting operations. }\end{array}$ \\
\hline \multirow{4}{*}{$\begin{array}{l}\text { Pied-billed Grebe* } \\
\text { (Podilymbus podiceps) }\end{array}$} & 1880 & Six nests containing eggs observed. \\
\hline & 1930 & Frequently seen, accompanied by well-grown young. \\
\hline & 1960 & Seven pairs, 3 nests, 2 broods of 5 to 6 young. \\
\hline & 2017 & Seven seen; known breeder. \\
\hline \multirow{4}{*}{$\begin{array}{l}\text { Pileated Woodpecker* } \\
\text { (Dryocopus pileatus) }\end{array}$} & 1880 & Not listed \\
\hline & 1930 & Not listed \\
\hline & 1960 & Not listed \\
\hline & 2017 & $\begin{array}{l}\text { Very rare. One seen in woodlot just west of the Locust Grove. Was } \\
\text { seen leaving the property flying north. }\end{array}$ \\
\hline \multirow{4}{*}{$\begin{array}{l}\text { Prothonotary Warbler* } \\
\text { (Protonotaria citrea) }\end{array}$} & 1880 & Not listed \\
\hline & 1930 & $\begin{array}{l}\text { A pair in damp wood at edge of the marsh near the clubhouse. One } \\
\text { of the birds carried food in its bill, inferentially for its young. }\end{array}$ \\
\hline & 1960 & Two nesting pairs in same woods. \\
\hline & 2017 & One seen on the banks of Green Creek. \\
\hline \multirow{4}{*}{$\begin{array}{l}\text { Purple Martin* } \\
\text { (Progne subis) }\end{array}$} & 1880 & Not listed \\
\hline & 1930 & Frequently seen in air; colonies in neighboring towns. \\
\hline & 1960 & $\begin{array}{l}\text { Estimated } 75 \text { pairs in the martin houses in yard and in eaves of } \\
\text { boat house. }\end{array}$ \\
\hline & 2017 & Forty-six seen; new martin houses installed this year. \\
\hline \multirow{4}{*}{$\begin{array}{l}\text { Red-breasted Merganser } \\
\text { (Mergus serrator) }\end{array}$} & 1880 & Identified in summer. \\
\hline & 1930 & Not found \\
\hline & 1960 & Not found; no recent summer records. \\
\hline & 2017 & Not found \\
\hline \multirow{4}{*}{$\begin{array}{l}\text { Red-bellied Woodpecker* } \\
\text { (Melanerpes carolinus) }\end{array}$} & 1880 & Not listed \\
\hline & 1930 & Not listed \\
\hline & 1960 & Not listed \\
\hline & 2017 & One seen \\
\hline
\end{tabular}


Table (continued)

\begin{tabular}{|c|c|c|}
\hline Species & Date & Notes \\
\hline \multirow[t]{4}{*}{$\begin{array}{l}\text { Red-eyed Vireo * } \\
\text { (Vireo olivaceus) }\end{array}$} & 1880 & $\begin{array}{l}\text { Much less numerous than in southwestern Ohio, being evidently } \\
\text { replaced to a great extent by the Warbling Vireo which was } \\
\text { exceedingly common. }\end{array}$ \\
\hline & 1930 & Would accept Dr. Langdon's statement. \\
\hline & 1960 & Two pairs seen. \\
\hline & 2017 & Not found \\
\hline \multirow[t]{4}{*}{$\begin{array}{l}\text { Redhead * } \\
\text { (Aythya americana) }\end{array}$} & 1880 & $\begin{array}{l}\text { One specimen, a male, taken June } 28,1879 \text {. (Evidently not seen } \\
\text { in } 1880 . \text { ) }\end{array}$ \\
\hline & 1930 & Not found \\
\hline & 1960 & Pair seen, probably non-breeding birds. \\
\hline & 2017 & Not found \\
\hline \multirow{4}{*}{$\begin{array}{l}\text { Red-headed Woodpecker* } \\
\text { (Melanerpes } \\
\text { erythrocephalus) }\end{array}$} & 1880 & Common \\
\hline & 1930 & Common \\
\hline & 1960 & $\begin{array}{l}\text { Two pairs seen; this species is probably much less abundant than } \\
\text { formerly. }\end{array}$ \\
\hline & 2017 & Not found; this species is present but not abundant. \\
\hline \multirow{4}{*}{$\begin{array}{l}\text { Red-tailed Hawk* } \\
\text { (Buteo jamaicensis) }\end{array}$} & 1880 & Not listed \\
\hline & 1930 & Not listed \\
\hline & 1960 & One seen \\
\hline & 2017 & Eight seen \\
\hline \multirow[t]{4}{*}{$\begin{array}{l}\text { Red-winged Blackbird * } \\
\text { (Agelaius phoeniceus) }\end{array}$} & 1880 & $\begin{array}{l}\text { As would be expected, the redwings were abundant in the marsh, } \\
\text { rising before us at every few yards from their nests. At the time of } \\
\text { our visit the young of the first brood were on the wing, and the } \\
\text { second set of eggs had evidently not yet been completed in most } \\
\text { cases, as most of the nests observed contained but } 2 \text { or } 3 \text { eggs } \\
\text { which were quite fresh. }\end{array}$ \\
\hline & 1930 & Very abundant. Dr. Langdon's notes apply to present conditions. \\
\hline & 1960 & Estimated 1,200 seen. Probably more abundant than in 1880 . \\
\hline & 2017 & $\begin{array}{l}\text { There were } 483 \text { seen; many more than that present. Very abundant } \\
\text { breeding species throughout summer. }\end{array}$ \\
\hline \multirow{4}{*}{$\begin{array}{l}\text { Ring-billed Gull * } \\
\text { (Larus delawarensis) }\end{array}$} & 1880 & Not listed \\
\hline & 1930 & Not listed \\
\hline & 1960 & Two seen \\
\hline & 2017 & Seven seen over the bay. \\
\hline \multirow{4}{*}{$\begin{array}{l}\text { Ring-necked Duck } \\
\text { (Aythya collaris) }\end{array}$} & 1880 & Not listed \\
\hline & 1930 & Not listed \\
\hline & 1960 & One male, apparently non-breeding bird. \\
\hline & 2017 & Not found \\
\hline
\end{tabular}


Table (continued)

\begin{tabular}{|c|c|c|}
\hline Species & Date & Notes \\
\hline \multirow{4}{*}{$\begin{array}{l}\text { Ring-necked Pheasant * } \\
\text { (Phasianus colchicus) }\end{array}$} & 1880 & Not listed \\
\hline & 1930 & Common at edges of woods bordering the swamp. \\
\hline & 1960 & $\begin{array}{l}\text { Seventeen adults plus } 3 \text { broods. The Mackey Piece, where pheasants } \\
\text { are very common, was not checked since it was not included in } \\
\text { previous censuses. }\end{array}$ \\
\hline & 2017 & Not found \\
\hline \multirow{4}{*}{$\begin{array}{l}\text { Ruby-throated } \\
\text { Hummingbird }{ }^{*} \\
\text { (Archilochus colubris) }\end{array}$} & 1880 & Not listed \\
\hline & 1930 & Not listed \\
\hline & 1960 & One pair seen regularly between dog kennels and woods. \\
\hline & 2017 & Not found on survey. Occasionally observed. \\
\hline \multirow{4}{*}{$\begin{array}{l}\text { Ruffed Grouse } \\
\text { (Bonasa umbellus) }\end{array}$} & 1880 & Rare \\
\hline & 1930 & Not found \\
\hline & 1960 & Not found \\
\hline & 2017 & Not found \\
\hline \multirow{4}{*}{$\begin{array}{l}\text { Sandhill Crane }{ }^{*} \\
\text { (Antigone canadensis) }\end{array}$} & 1880 & Not listed \\
\hline & 1930 & Not listed \\
\hline & 1960 & Not listed \\
\hline & 2017 & $\begin{array}{l}\text { Two seen; flying out of Hopfinger's west of Darr. Nested in Ottawa } \\
\text { Shooting Club near Dry Bunch in } 2016 \text {. }\end{array}$ \\
\hline \multirow{4}{*}{$\begin{array}{l}\text { Savannah Sparrow * } \\
\text { (Passerculus sandwichensis) }\end{array}$} & 1880 & Not listed \\
\hline & 1930 & $\begin{array}{l}\text { Common in boggy pasturelands between Winous Point and the } \\
\text { highway. }\end{array}$ \\
\hline & 1960 & Nine seen. Status probably unchanged since 1930. \\
\hline & 2017 & Not found \\
\hline \multirow{4}{*}{$\begin{array}{l}\text { Semipalmated Sandpiper } \\
\text { (Calidris pusilla) }\end{array}$} & 1880 & Not listed \\
\hline & 1930 & Not listed \\
\hline & 1960 & Not listed \\
\hline & 2017 & $\begin{array}{l}\text { Not observed. Common, have banded over 3,000 in summer } \\
\text { banding from } 2006 \text { to } 2015 \text {. }\end{array}$ \\
\hline \multirow{4}{*}{$\begin{array}{l}\text { Sharp-shinned Hawk } \\
\text { (Accipiter striatus) }\end{array}$} & 1880 & May 1877 \\
\hline & 1930 & Not found \\
\hline & 1960 & Not found \\
\hline & 2017 & Not observed but known to be a summer resident. \\
\hline
\end{tabular}


Table (continued)

\begin{tabular}{|c|c|c|}
\hline Species & Date & Notes \\
\hline \multirow{4}{*}{$\begin{array}{l}\text { Short-billed Dowitcher } \\
\text { (Limnodromus griseus) }\end{array}$} & 1880 & Not listed \\
\hline & 1930 & Not listed \\
\hline & 1960 & Not listed \\
\hline & 2017 & $\begin{array}{l}\text { Seven seen wading and feeding in southeast corner of Horseshoe; } \\
799 \text { banded from } 2006 \text { to } 2015 \text { in the summer months. }\end{array}$ \\
\hline \multirow{4}{*}{$\begin{array}{l}\text { Snowy Egret } \\
\text { (Egretta thula) }\end{array}$} & 1880 & Not listed \\
\hline & 1930 & Not listed \\
\hline & 1960 & Not listed \\
\hline & 2017 & $\begin{array}{l}\text { None observed during survey. Rarely but occasionally observed in } \\
\text { early summer. }\end{array}$ \\
\hline \multirow{4}{*}{$\begin{array}{l}\text { Solitary Sandpiper } \\
\text { (Tringa solitaria) }\end{array}$} & 1880 & July 1879 to 1880 \\
\hline & 1930 & Not found \\
\hline & 1960 & Not found \\
\hline & 2017 & Not found \\
\hline \multirow{4}{*}{$\begin{array}{l}\text { Song Sparrow* } \\
\text { (Melospiza melodia) }\end{array}$} & 1880 & Common \\
\hline & 1930 & Common \\
\hline & 1960 & Forty-seven seen; by far the dominant sparrow around the marsh. \\
\hline & 2017 & Sixty-four seen; observations concur with the 1960 survey. \\
\hline \multirow{4}{*}{$\begin{array}{l}\text { Sora } * \\
(\text { Porzana carolina })\end{array}$} & 1880 & Not listed \\
\hline & 1930 & Identified once by its high double-noted call. \\
\hline & 1960 & One seen \\
\hline & 2017 & $\begin{array}{l}\text { Frequently heard and observed during spring and summer } \\
\text { marshbird surveys, known to nest, likely nests less frequently } \\
\text { than Virginia Rails. Banded } 128 \text { spring migrants since } 2015 .\end{array}$ \\
\hline \multirow{4}{*}{$\begin{array}{l}\text { Spotted Sandpiper * } \\
\text { (Actitis macularius) }\end{array}$} & 1880 & Common \\
\hline & 1930 & Common \\
\hline & 1960 & Twenty-six seen \\
\hline & 2017 & Four seen \\
\hline \multirow{4}{*}{$\begin{array}{l}\text { Swamp Sparrow }{ }^{*} \\
(\text { Melospiza georgiana })\end{array}$} & 1880 & Not listed \\
\hline & 1930 & Not listed \\
\hline & 1960 & Not listed \\
\hline & 2017 & Three heard; probably under represented. \\
\hline
\end{tabular}


Table (continued)

\begin{tabular}{|c|c|c|}
\hline Species & Date & Notes \\
\hline \multirow[t]{4}{*}{$\begin{array}{l}\text { Tree Swallow * } \\
\text { (Tachycineta bicolor) }\end{array}$} & 1880 & $\begin{array}{l}\text { An abundant species; the prevailing swallow of the locality. } \\
\text { Numerous young of the first brood observed on the wing in } \\
\text { their peculiarly silver drab first plumage. On June } 29 \text { th, a nest } \\
\text { containing one egg and a young bird (probably of the second } \\
\text { brood) was observed. }\end{array}$ \\
\hline & 1930 & Present but not abundant. \\
\hline & 1960 & $\begin{array}{l}\text { At least } 98 \text { seen along lane and adjacent marsh. Still the prevailing } \\
\text { swallow of the locality. }\end{array}$ \\
\hline & 2017 & $\begin{array}{l}\text { Three hundred and five seen. The most abundant breeding } \\
\text { swallow at WPMC. }\end{array}$ \\
\hline \multirow{4}{*}{$\begin{array}{l}\text { Trumpeter Swan * } \\
\text { (Cygnus buccinator) }\end{array}$} & 1880 & Not listed \\
\hline & 1930 & Not listed \\
\hline & 1960 & Not listed \\
\hline & 2017 & $\begin{array}{l}\text { Estimated } 4 \text { to } 6 \text { breeding pairs on Winous Point. Introduced in } \\
\text { the 1990s. }\end{array}$ \\
\hline \multirow{4}{*}{$\begin{array}{l}\text { Tufted Titmouse * } \\
\text { (Baeolophus bicolor) }\end{array}$} & 1880 & Not listed \\
\hline & 1930 & Not listed \\
\hline & 1960 & Not listed \\
\hline & 2017 & One seen \\
\hline \multirow{4}{*}{$\begin{array}{l}\text { Turkey Vulture* } \\
\text { (Cathartes aura) }\end{array}$} & 1880 & Not listed \\
\hline & 1930 & Not listed \\
\hline & 1960 & One seen \\
\hline & 2017 & Two seen flying over fields north of North Lily. \\
\hline \multirow{4}{*}{$\begin{array}{l}\text { Upland Sandpiper* } \\
\text { (Bartramia longicauda) }\end{array}$} & 1880 & Not listed \\
\hline & 1930 & One seen in stubble field, a mile to the north of Winous Point. \\
\hline & 1960 & $\begin{array}{l}\text { Not found. No recent summer records; regular spring visitor, } \\
\text { probably breeds in fields adjacent to marsh. }\end{array}$ \\
\hline & 2017 & Not found \\
\hline \multirow{4}{*}{$\begin{array}{l}\text { Virginia Rail * } \\
\text { (Rallus limicola) }\end{array}$} & 1880 & Not listed \\
\hline & 1930 & $\begin{array}{l}\text { One seen. A note believed to be theirs frequently heard. Generally } \\
\text { regarded as common. }\end{array}$ \\
\hline & 1960 & One seen \\
\hline & 2017 & $\begin{array}{l}\text { Frequently heard and observed during spring and summer } \\
\text { marshbird surveys, known to nest, although uncertainty about } \\
\text { how common nesting is at WPMC. Banded } 342 \text { spring migrants } \\
\text { since } 2015 \text {. }\end{array}$ \\
\hline
\end{tabular}


Table (continued)

\begin{tabular}{|c|c|c|}
\hline Species & Date & Notes \\
\hline \multirow[t]{4}{*}{$\begin{array}{l}\text { Warbling Vireo * } \\
\text { (Vireo gilvus) }\end{array}$} & 1880 & $\begin{array}{l}\text { As above stated, this species was found in considerable numbers, } \\
\text { the woods and scattered groves on the borders of the marsh } \\
\text { resounding with their notes. }\end{array}$ \\
\hline & 1930 & Would accept Dr. Langdon's statements. \\
\hline & 1960 & $\begin{array}{l}\text { Nine seen. Perhaps disappearance of elms is causing decline of } \\
\text { this species. }\end{array}$ \\
\hline & 2017 & Three seen in Mackey woods. \\
\hline \multirow{4}{*}{$\begin{array}{l}\text { Wild Turkey* } \\
(\text { Meleagris gallopavo) }\end{array}$} & 1880 & Rare \\
\hline & 1930 & Not found \\
\hline & 1960 & Not found \\
\hline & 2017 & Not found, but previously, albeit rarely, observed in last ten years. \\
\hline \multirow{4}{*}{$\begin{array}{l}\text { Willow Flycatcher* } \\
\text { (Empidonax traillii) }\end{array}$} & 1880 & Not listed \\
\hline & 1930 & Not listed \\
\hline & 1960 & Eight seen; nests in thickets along ditches. \\
\hline & 2017 & $\begin{array}{l}\text { Fourteen seen; often heard before visually observed. Can be found } \\
\text { in thick shrubbery lining the dikes. }\end{array}$ \\
\hline \multirow{4}{*}{$\begin{array}{l}\text { Wilson's Snipe * } \\
\text { (Gallinago delicata) }\end{array}$} & 1880 & Several shot in July 1878. \\
\hline & 1930 & Not found \\
\hline & 1960 & One seen \\
\hline & 2017 & Not found. Common fall migrant. \\
\hline \multirow{4}{*}{$\begin{array}{l}\text { Wood Duck* } \\
\text { (Aix sponsa) }\end{array}$} & 1880 & Not listed \\
\hline & 1930 & $\begin{array}{l}\text { The duck most abundantly seen in the marsh. We surmised several } \\
\text { family parties in the sheltered bays amid the cattails. In one case the } \\
\text { young, unable to fly, scurried away through the stalks. }\end{array}$ \\
\hline & 1960 & Thirty-two seen; four nests in boxes around clubhouse. \\
\hline & 2017 & $\begin{array}{l}\text { Common summer breeder, ducklings observed frequently. We have } \\
\text { summer banded 1,291 individuals since } 2011 \text {. }\end{array}$ \\
\hline \multirow{4}{*}{$\begin{array}{l}\text { Wood Thrush * } \\
\text { (Hylocichla mustelina) }\end{array}$} & 1880 & A few observed in the groves bordering the marsh. \\
\hline & 1930 & Not found \\
\hline & 1960 & Not found \\
\hline & 2017 & Not found \\
\hline \multirow{4}{*}{$\begin{array}{l}\text { Yellow-breasted Chat } * \\
\text { (Icteria virens) }\end{array}$} & 1880 & Not listed \\
\hline & 1930 & One pair in open, bush-grown, dry woods near the clubhouse. \\
\hline & 1960 & Not found \\
\hline & 2017 & Not found \\
\hline
\end{tabular}


Table (continued)

\begin{tabular}{|c|c|c|}
\hline Species & Date & Notes \\
\hline \multirow[t]{4}{*}{$\begin{array}{l}\text { Yellow Warbler }{ }^{*} \\
\text { (Setophaga petechia) }\end{array}$} & 1880 & $\begin{array}{l}\text { A very common species in the woods bordering the marsh; young } \\
\text { of the first brood observed on the wing. }\end{array}$ \\
\hline & 1930 & Would accept Dr. Langdon's statement. \\
\hline & 1960 & Twenty-nine seen \\
\hline & 2017 & $\begin{array}{l}\text { Sixty-seven seen; by far the most common warbler on the property } \\
\text { during the summer. }\end{array}$ \\
\hline \multirow{4}{*}{$\begin{array}{l}\text { Yellow-billed Cuckoo* } \\
\text { (Coccyzus americanus) }\end{array}$} & 1880 & Not listed \\
\hline & 1930 & $\begin{array}{l}\text { Pair noted, calling constantly about borders of wood adjacent to } \\
\text { clubhouse. }\end{array}$ \\
\hline & 1960 & Six seen. Common nester along lane and around clubhouse. \\
\hline & 2017 & Three seen; uncommon. Seen mostly in shrubbery or trees along dikes. \\
\hline \multirow{4}{*}{$\begin{array}{l}\text { Yellow-headed Blackbird * } \\
\text { (Xanthocephalus } \\
\text { xanthocephalus) }\end{array}$} & 1880 & Not listed \\
\hline & 1930 & Not listed \\
\hline & 1960 & $\begin{array}{l}\text { One, possibly two, birds seen around clubhouse since June } 8 \text {, } \\
\text { 1960. One appears to be a female, the other a sub-adult male. This } \\
\text { appears to be the first record for the Sandusky Bay Area. Neither } \\
\text { specimen has been seen since July } 8,1960 \text {. }\end{array}$ \\
\hline & 2017 & Not found, only observed once at any time of year since 2008 . \\
\hline
\end{tabular}




\section{LITERATURE CITED}

American Ornithologists' Union. 1998. Check-list of North American birds: the species of birds of North America from the Arctic through Panama, including the West Indies and Hawaiian Islands. 7th ed. Washington (DC): American Ornithologists' Union. 829 p. ISBN13: 978-1891276002.

Anders AD, Faaborg J, Thompson FR 3rd. 1998. Postfledging dispersal, habitat use, and home-range size of juvenile Wood Thrushes. Auk. 115(2):349-358.

https://doi.org/10.2307/4089193

Anderson JM. 1960. Summer birds of Winous Point in 1880, 1930, and 1960. Winous Point Shooting Club. Unpublished report. 20 p.

Andrews R. 1952. A study of waterfowl nesting on a Lake Erie marsh [master's thesis]. [Columbus $(\mathrm{OH})$ ]: The Ohio State University.

Ball LC, Doherty DF Jr, McDonald MW. 2005. An occupancy modeling approach to evaluating a Palm Springs ground squirrel habitat model. J Wildlife Manage. 69(3):894-904. https://doi.org/10.2193/0022-541X(2005)069[0894:AOM ATE]2.0.CO;2

Brennan LA, Kuvlesky WP Jr. 2005. Invited paper: North American grassland birds: an unfolding conservation crisis? J Wildlife Manage. 69(1):1-13.

https://doi.org/10.2193/0022-541X(2005)069<0001:NAG $\mathrm{BAU}>2.0 . \mathrm{CO} ; 2$

Campbell LW, Gavin C, Mayfield H. 1995. The marshes of southwestern Lake Erie. Athens $(\mathrm{OH})$ : Ohio University Press. 231 p. ISBN13: 978-0821410943.

Christy BH. 1931. A fifty-year comparison. Auk. 48(3):365-378. https://doi.org/10.2307/4076481

Franklin JF. 1989. Importance and justification of long-term studies in ecology. In: Likens GE, editor. Long-term studies in ecology. New York (NY): Springer-Verlag. p. 3-19. https://doi.org/10.1007/978-1-4615-7358-6_1

Grier JW. 1982. Ban of DDT and subsequent recovery of reproduction in Bald Eagles. Science. 218(4578):1232-1235. https://doi.org/10.1126/science.7146905

Heckert JR. 1995. An analysis of midwestern breeding bird population trends: 1966-1993. Am Midl Nat. 134(1):41-50. https://doi.org/10.2307/2426481

Jackson DA, Somers KM, Harvey HH. 1989. Similarity coefficients: measures of co-occurrence and association or simply measures of occurrence? Am Nat. 133(3):436-453. https://doi.org/10.1086/284927

Langdon FW. 1880. Summer birds of a northern Ohio marsh. J Cincinnati Soc Nat Hist. 3(3):220-232. https://biodiversitylibrary.org/page/7313762

Maxson SJ, Fieberg JR, Riggs MR. 2007. Black Tern nest habitat selection and factors affecting nest success in northwestern Minnesota. Waterbirds. 30(1):1-9.

https://doi.org/10.1675/1524-4695(2007)030[0001:BTN HSA]2.0.CO;2
[ODNR] Ohio Department of Natural Resources, Division of Wildlife. 2018. Trumpeter Swan. [Accessed 2018 Nov 8]. http://wildlife.ohiodnr.gov/species-and-habitats/speciesguide-index/birds/trumpeter-swan

Peterjohn BG, Rice DL. 1991. The Ohio breeding bird atlas. Columbus (OH): Ohio Department of Natural Resources, Division of Natural Areas and Preserves. 416 p.

ISBN13: 978-0931079047. https://hdl.handle.net/2027/coo.31924101927600

Rodewald PG, Shumar MB, Boone AT, Slager DL, McCormac J, editors. 2016. The second atlas of breeding birds in Ohio. University Park (PA): The Pennsylvania State University Press. 578 p. ISBN13: 978-0271071275.

Rollins D, Carroll JP. 2001. Impacts of predation on Northern Bobwhite and Scaled Quail. Wildlife Soc B. 29(1):39-51. https://www.jstor.org/stable/3783979

Sauer JR, Link WA, Fallon JE, Pardieck KL, Ziolkowski DJ Jr. 2013. The North American breeding bird survey 1966-2011: summary analysis and species accounts. N Amer Fauna. 79:1-32.

https://doi.org/10.3996/nafa.79.0001

Sedgwick T, Kroll R. 2010. Winous Point: 150 years of waterfowling and conservation. Lanham (MD): The Derrydale Press. 333 p. ISBN13: 978-1564162144.

Sherman DE, Kroll RW, Engle TL. 1996. Flora of a diked and an undiked southwestern Lake Erie wetland. Ohio J Sci. 96(1):4-8. http://hdl.handle.net/1811/23690

Smith GW (U.S. Fish and Wildlife Service, Laurel, MD). 1995. A critical review of the aerial and ground surveys of breeding waterfowl in North America. Washington (DC): U.S. Department of the Interior, National Biological Service. 252 p. Biological Science Report 5. Available from: NTIS, Springfield, VA; PB96-121876.

Thompson SA, Price JJ. 2006. Water clarity and diving behavior in wintering Common Loons. Waterbirds. 29(2):169-175. https://www.jstor.org/stable/4132565

Vermeer K. 1973. Some aspects of the nesting requirements of Common Loons in Alberta. Wilson Bull. 85(4):429-435.

Wilson RF, Mitsch WJ. 1996. Functional assessment of five wetlands constructed to mitigate wetland loss in Ohio, USA. Wetlands. 16(4):436-451. https://doi.org/10.1007/BF03161333

Wintle BA, Bardos DC. 2006. Modeling species-habitat relationships with spatially autocorrelated observational data. Ecol Appl. 16(5):1945-1958. https://doi.org/10.1890/1051-0761(2006)016[1945:MSR WSA] 2.0.CO;2 